\title{
Application of ESI FT-ICR MS to Study Kraft Lignin Modification by the Exoenzymes of the White Rot Basidiomycete Fungus TrametesHirsutaLE-BIN 072

\author{
Konstantin V.Moiseenko, Olga S.Savinova, Olga A.Glazunova, Arkadiy P. \\ Sinitsyn, and Tatiana V. Fedorova
}

A. N. Bach Institute of Biochemistry, Research Center of Biotechnology, Russian Academy of Sciences, Leninsky Ave. 33/2, Moscow 119071, Russia

ORCID

Konstantin Moiseenko; 0000-0002-3335-3641

Corresponding Author:

Konstantin Moiseenko; email:

mr.moiseenko@gmail.com

\section{Dates}

Published 13 January 2022

Publishing services provided by

Knowledge E

(c) Konstantin V.Moiseenko et

al. This article is distributed

under the terms of the

Commons Attribution License,

which permits unrestricted use and redistribution provided that the original author and source are credited.

Selection and Peer-review under the responsibility of the 8th Scientific and Practical Conference Conference Committee.

\section{G OPEN ACCESS}

Abstract. Trameteshirsuta is a wood rotting fungus that possesses a vast array of lignin degrading enzymes, including7 laccases, 7 ligninolyticmanganese peroxidases, 9 lignin peroxidases and 2 versatile peroxidases. In this study,electrospray ionization Fourier transform ion cyclotron resonance mass spectrometry (ESI FT-ICR MS)was used to examine kraft lignin modification by the enzymatic system of this fungus.The observed pattern of lignin modification suggested that before the $6^{\text {th }}$ day of cultivation,the fungal enzymatic system tended to degrade more oxidized moleculesand, hence, less recalcitrant molecules, with the production of hard-to-modify reduced molecular species. At some point after the $6^{\text {th }}$ day of cultivation,the fungus started to degrade less oxidized, more recalcitrant, compounds, converting them into the more oxidized forms. The altered pattern of lignin modification enabled changes in the fungal enzymatic system. These changes were further attributed to the appearance of the particular ligninolyticmanganese peroxides enzyme(MnP7), which was added by the fungus to the mixture of enzymes that had already been secreted (VP2 and MnP5).

Keywords: wood rotting fungi, kraft lignin, mass spectrometry, peroxidases

\section{Introduction}

Lignin is one of the three major components of the cellular wall invascularplantsand the second most abundant, after cellulose, biopolymer on Earth [1]. Being a complex polymeric molecule composed of aromatic units (monolignols), lignin represents an indispensable source of many phenolic compounds [2]. Although irregular covalent linkage of monolignols made lignin extremely chemically recalcitrant, it can be efficiently degraded by a particular type of wood rotting fungi - white rots. To efficiently degrade lignin, white rotting fungi poses two main group of enzymes - laccases and class II (ligninolytic) peroxidases [3]. The group of ligninolytic peroxidases is further subdivided into manganese peroxidases (MnPs), lignin peroxidases (LiPs) and versatile peroxidases (VPs) [4]. Virtually all white-rot fungi contain several non-allele copies of laccase and 
peroxidase genes in their genomes; however, neither their regulation nor particular role in the process of lignin degradation are known $[5,6]$.

Apart from understudied enzymatic system,the major challenge in the investigation of lignin modification by white rotting fungi is characterization of individual compounds produced in this process. Unfortunately, both classical methods of wet chemistry and modern analytical spectroscopic methods (e.g. UV/FTIR/Raman spectroscopies and NMR) can only provide crude estimates of average structures and functional groups in lignin $[7,8]$. On the other hand, recently emerging technic of electrospray ionization Fourier transform ion cyclotron resonance mass spectrometry (ESI FT-ICR MS) allows to perform a compound-wise full scan analysis of a lignin sample. Due to its enormous resolution power, FT-ICR MS can simultaneously detect thousands of lignin degradation products, to which elemental molecular formulas can be further assigned $[9,10]$.

Trameteshirsuta is a typical representative of the white root fungi, which possesses 7 laccase and 18 ligninolytic peroxidase (7 - MnPs, 9 - LiPs and 2 - VPs,) genes in its genome[5, 6,11$]$. In this article the process of kraft lignin modification by $T$. hirsuta enzymatic system was studied using (-)ESI FT-ICR MS method and particular enzymes involved in this process were determined by 2D gel electrophoresis followed by matrix-assisted laser desorption/ionization time-of-flight/time-of-flight mass spectrometry (MALDI-TOF/TOF MS) technique.

\section{Materials and Methods}

The fungal strain Trameteshirsuta LE-BIN 072 was obtained from the Komarov Botanical Institute Basidiomycetes Culture Collection (LE-BIN; St. Petersburg, Russia). The sequence of its ITS1-5.8S rRNA-ITS2 region is available at the NCBI GenBank accession AB158313, and the whole-genome sequence - GCA_001302255.2.

In the laboratory fungal mycelium was stored on wort-agar slants at $4{ }^{\circ} \mathrm{C}$. To obtain starting inoculum, fungus was cultivated in $750 \mathrm{~mL}$ Erlenmeyer flasks with $200 \mathrm{~mL}$ of glucose-peptone (GP) medium (per $1 \mathrm{~L}$ of $\mathrm{dH} 2 \mathrm{O}$ ): $3.0 \mathrm{~g}$ peptone, $10.0 \mathrm{~g}$ glucose, $0.6 \mathrm{~g} \mathrm{KH}_{2} \mathrm{PO}_{4}, 0.4 \mathrm{~g} \mathrm{~K}_{2} \mathrm{HPO}_{4}, 0.5 \mathrm{~g} \mathrm{MgSO}_{4}, 50 \mathrm{mg} \mathrm{MnSO}$, $1 \mathrm{mg} \mathrm{ZnSO}$, and $0.5 \mathrm{mg}$ $\mathrm{FeSO}_{4}$. The cultivation was carried out statically in the dark at $26-28{ }^{\circ} \mathrm{C}$. The inoculum was obtained by disruption of the mycelium with ceramic beads; allinoculationswere performed with $25 \mathrm{ml}$ of disrupted mycelium.

For lignin degradation experiment, the $200 \mathrm{~mL}$ of GP medium was supplied with the $2 \mathrm{~g} / \mathrm{l}$ of alkali lignin (Sigma-Aldrich, USA). The submerged cultivation was performed 
on Brunswick Innova 44 (Eppendorf Inc., USA) rotary shaker at 180 RPM in the dark at $26-28^{\circ} \mathrm{C}$

Preparation of lignin samples and (-)ESI FT-ICR MS was performed as described in [12]. Preparation of exoproteomic samples, 2D gel electrophoresis and MALDITOF/TOFMSwas performed as described in[13].

\section{Result and Discussion}

As a result of (-)ESI FT-ICR MS analysis, 5999, 5382 and 6134 different compounds were identified in intact kraft lignin (control) sample and lignin samples collected after the 6th and 10th days of fungal cultivation, respectively. In all the samples, the observed molecular weights of the compounds were distributed on the interval between 200 and $700 \mathrm{Da}$.In the course of the experiment the mean molecular masses of the compounds decreased. In the control sample molecular masses of the compounds were $470 \pm 117 \mathrm{Da}$, while in the samples collected after the 6th and 10th days of fungal cultivationthey comprised $458 \pm 117 \mathrm{Da}$ and $446 \pm 118 \mathrm{Da}$, respectively.

Since kraft lignin is a heterogeneous mixture of many polymeric compounds, the data obtained by (-)ESI FT-ICR MS inheritably contained a certain amount of random noise that preventedobservation of systematical changes occurred dew to the action of fungus. To denoise the data, all the determined compounds were classified into the 6groups (Figure 1):AlwaysPresent group contained compounds that were present in all the samples; LostByDay6 and LostByDay10 groups contained compounds that were present until the 6th and 10th days of fungal cultivation, respectively; GainOnDay6 and GainOnDay10 groups contained compounds that were constantly present in the samples starting from the6th and 10th days of fungal cultivation, respectively; Miscel group contained compounds that were either present only on the 6th day of cultivation or simultaneously present in the control and on the 10th day of cultivation.

With respect to the fungal modification of kraft lignin the mostinformative groups wereLostByDay6,LostByDay10,GainOnDay6 and GainOnDay10. While the former two contained compounds that was systematically degraded by fungal enzymatic system, the latter two contained compounds that once formed presumably cannot be further modified by the fungus. To display general compositional characteristics of these groups the panel ofthe $\mathrm{H} / \mathrm{C}$ versus $\mathrm{O} / \mathrm{C}$ elementalratiosplots- the van Krevelen diagrams was constructed (Figure 2). Different typesofmoleculesoccupiesdifferent regions ofthevanKrevelen diagram [14]; previously,these regions were named according to their most representative compounds[15]. To partially alleviate such well known problem of 

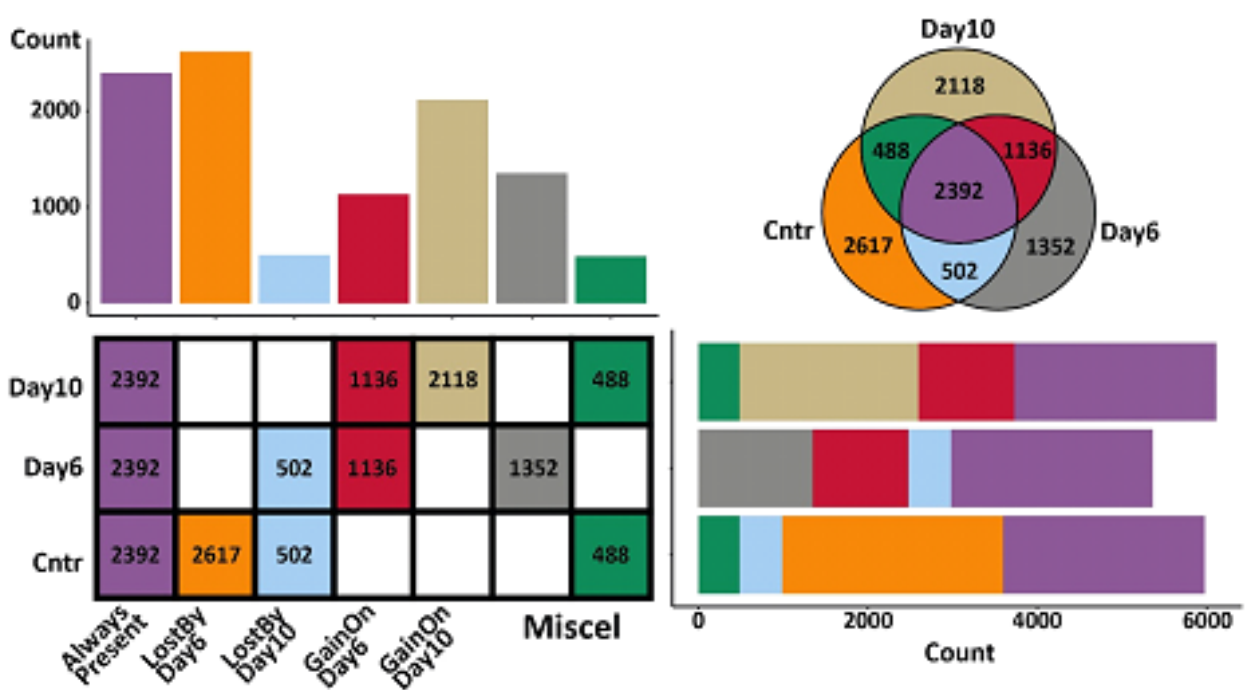

Figure 1: The UpSet plot and Venn diagram depicting the partitioning of the compounds.
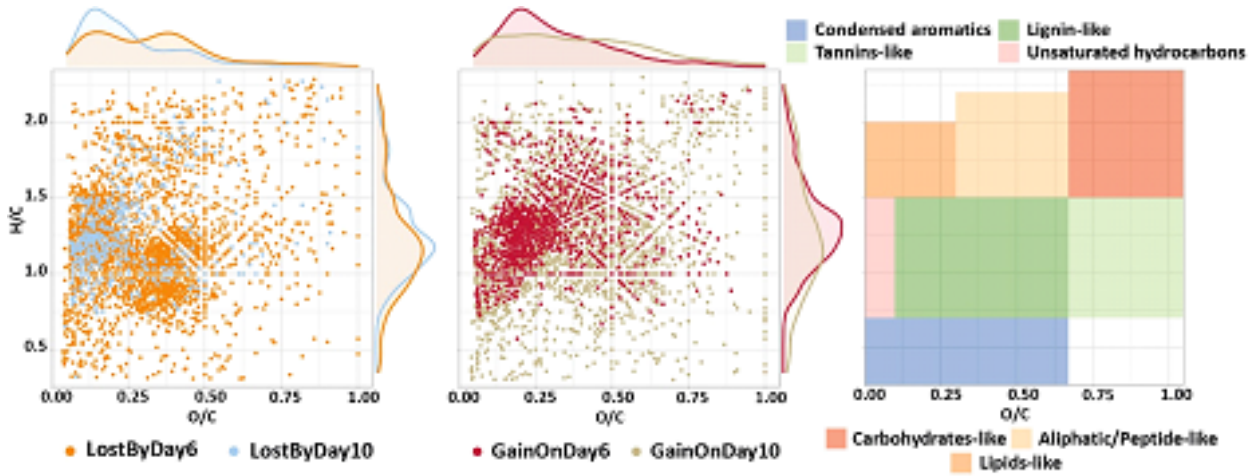

Figure 2: The van Krevelen diagrams for the selected groups of compounds.

vanKrevelendiagramsasoverplotting of molecules with different formulas but the same $\mathrm{H} / \mathrm{C}$ and $\mathrm{O} / \mathrm{C}$ ratios [16], the marginal distributions were added to each axes of the plots.

As it can be seen from the van Krevelen diagrams (Figure 2), the compounds from LostByDay6 group mostly liednear the center of the lignin-like area followed by the unsaturated hydrocarbons area. In contrast, the compounds from LostByDay10 group predominantly occupied unsaturated hydrocarbons area and its boundary with the lignin-like area; the minority of compounds lied near the center of the lignin-like area. Interestingly, in case of theGainOnDay6 and GainOnDay10groups, the opposite situation was observed: the compounds from the GainOnDay6group lied either in the unsaturated hydrocarbons area or in its close proximity, while the compounds from the GainOnDay10group tended to spread near the center of the lignin-like area.

In general, the observed changes in the distribution of compounds suggested that before the 6th day of cultivation fungal enzymatic system tended to degrade more oxidized and, hence, less recalcitrant molecules with production of hardto modify reduced 
molecular species. At some point after the 6th day fungus started to degrade less oxidized, more recalcitrant, compounds, converting them into the more oxidized one.

The observed pattern of the kraft lignin modification by the exoenzymes of $T$. hirsutasuggested changes in the fungal enzymatic system between the 6th and 10th days of its cultivation. Indeed, exoproteomic study demonstrated that: on the 6th day of cultivation only VP2 and MnP5 ligninolytic enzymes were secreted by the fungus; however, on the 10th day of cultivation the new enzyme - MnP7 - appeared.Hence, different patterns of lignin modification were not a result of a complete change of enzymatic system but the addition of the new enzyme to the already secreted ones.

\section{Conclusion}

The present article demonstrated applicability of the (-)ESI FT-ICR MS technique for studying lignin modification process by fungal enzymatic system. The compound-wise orientation of this technique allowed describing particular changes in the lignin composition during the fungal cultivation. Moreover, the changingpattern of the lignin modification allowed suggesting changes in the fungal enzymatic system in the process. This changes were further attributed to the appearance of the particular ligninolytic peroxidase $-\mathrm{MnP7}$.

\section{References}

[1] Ponnusamy VK, Nguyen DD et al. A review on lignin structure, pretreatments, fermentation reactions and biorefinery potential. Bioresource Technology. 2019;271:462472.

[2] Pandey MP, Kim CS. Lignin Depolymerization and conversion: A review of thermochemical methods. Chemical Engineering \& Technology. 2011;34(1):29-41.

[3] Dashtban M, Schraft H, Syed TA, Qin W. (2010). Fungal biodegradation and enzymatic modification of lignin. International Journal of Biochemistry and Molecular Biology. 2010;1(1):36-50.

[4] Hammel K, Cullen. D. Role of fungal peroxidases in biological ligninolysis. Current Opinion in Plant Biology. 2008;11(3):349-355.

[5] Vasina DV, Moiseenko KV, Fedorova TV, Tyazhelova TV. (2017). Lignin-degrading peroxidases in white-rot fungus Trameteshirsuta 072. Absolute expression quantification of full multigene family. PLOS ONE. 2017;12(3):e0173813- e0173814. 
[6] Moiseenko KV, Vasina DV,Farukshina KT,et al. Orchestration of the expression of the laccase multigene family in white-rot basidiomycete Trameteshirsuta 072: Evidences of transcription level subfunctionalization. Fungal Biology. 2018;122(5):353-362.

[7] Stark NM, Yelle DJ, Agarwal UP. (2016). Techniques for characterizing lignin, lignin in polymer composites. Amsterdam, Netherlands Elsevier; 2016.

[8] Lupoi JS, Singh S, Parthasarathi R, Simmons BA, Henry RJ. Recent innovations in analytical methods for the qualitative and quantitative assessment of lignin. Renewable and Sustainable Energy Reviews. 2015;49:871-906.

[9] Qi Y, Fu P, Li S, Ma C, Liu C, Volmer DA. Assessment of molecular diversity of lignin products by various ionization techniques and high-resolution mass spectrometry. Science of The Total Environment. 2020;713:136573-136573.

[10] Banoub J, Delmas G.-H., Joly N,et al. (2015). A critique on the structural analysis of lignins and application of novel tandem mass spectrometric strategies to determine lignin sequencing. Journal of Mass Spectrometry. 2015;50(1):5-48.

[11] Savinova OS, Moiseenko KV, Vavilova EA, et al. (2019). Evolutionary relationships between the laccase genes of polyporales: Orthology-based classification of laccase isozymes and functional insight from Trametes hirsute. Frontiers in Microbiology. 2019;10. 152-160 doi:10.3389/fmicb.2019.00152

[12] Zherebker A,Shirshin E,Rubekina A,et al. (2020). Optical properties of soil dissolved organic matter are related to acidic functions of its components as revealed by fractionation, selective deuteromethylation, and ultrahigh resolution mass spectrometry. Environmental Science \& Technology. 2020;54(5):2667-2677. doi:10.1021/acs.est.9b05298

[13] Vasina DV, Pavlov AR, Koroleva OV. Extracellular proteins of Trameteshirsutast. 072 induced by copper ions and a lignocellulose substrate. BMC Microbiology. 2016;16(1):106-110. doi:10.1186/s12866-016-0729-0

[14] Hertkorn N, Frommberger M, Witt M, Koch BP, Schmitt-Kopplin P, PerdueEM. Natural organic matter and the event horizon of mass spectrometry. Analytical Chemistry. 2008;80(23):8908-8919. doi:10.1021/ac800464g

[15] Rivas-Ubach A, Liu Y, Bianchi TS, TolićN, Jansson C, Paša-Tolić L. Moving beyond the van krevelen diagram: A new stoichiometric approach for compound classification in organisms. Analytical Chemistry. 2018;90(10):6152-6160. doi:10.1021/acs.analchem.8b00529

[16] Reemtsma T. Determination of molecular formulas of natural organic matter molecules by (ultra-) high-resolution mass spectrometry. Journal of Chromatography A. 2009;1216(18):3687-3701. doi:10.1016/j.chroma.2009.02.033 